\title{
El potencial de las sociedades de cazadores como herramienta de conservación en España
}

\author{
J.E. Gutiérrez $1, *$ \\ (1) SEO/BirdLife. Delegación de Andalucía. Calle Miguel Bravo Ferrer, 25 bajo, 41005 Sevilla, España. \\ * Autor de correspondencia: J.E. Gutiérrez [jegutierrez@seo.org]
}

> Recibido el 12 de febrero de 2013, aceptado el 29 de mayo de 2013.

Gutiérrez, J.E. (2013). El potencial de las sociedades de cazadores como herramienta de conservación en España. Ecosistemas 22(2):104106. Doi.: 10.7818/ECOS.2013.22-2.15

Los modelos de conservación "de arriba hacia abajo" se han mostrado insuficientes para frenar de manera efectiva la pérdida de biodiversidad. A raíz de este problema, fundamentalmente en países de África, Sudamérica y Asia, se han implementado programas basados en la participación de las comunidades locales en la conservación de sus territorios. Estos programas, basados en modelos "de abajo hacia arriba", han revelado la importancia de los procesos participativos para el éxito de los programas de conservación. Sin embargo en Europa, en donde muchos problemas de conservación tienen que ver con la falta de participación e implicación de los colectivos rurales, las iniciativas para incorporar procesos participativos son escasas y generalmente poco exitosas, siendo ineludible avanzar en la búsqueda de estrategias y herramientas eficaces para lograr este objetivo. En este contexto, las sociedades de cazadores en España podrían suponer una experiencia paradigmática de este modelo de conservación de "abajo hacia arriba". La gran superficie sobre la que tienen derecho de gestión, más de 6.5 millones de ha, buena parte de ella con un alto valor de conservación, su implantación en el medio rural y su modelo social, participativo y democrático en la toma de decisiones, las dota en su conjunto de un extraordinario potencial como herramienta de conservación. A pesar de ello, existe un gran desconocimiento de este modelo de aprovechamiento y de los efectos de sus externalidades ambientales. Avanzar en este sentido abriría una línea de investigación innovadora y de gran valor para la conservación de la biodiversidad en Europa.

Palabras clave: caza, colectivos rurales, usos comunales, conservación "de abajo hacia arriba".

Gutiérrez, J.E. (2013). The potential of hunting societies as a conservation tool in Spain. Ecosistemas 22(2):104-106. Doi.: 10.7818/ECOS.2013.22-2.15

"Top-down" conservation models have proved insufficient to effectively halt the loss of biodiversity. Because of this problem, programs based on participation of local communities in the conservation of their territories have been implemented, mainly in Africa, South America and Asia. These programs based on "bottom-up" models have revealed the importance of participatory processes for successful conservation programs. But in Europe, where many conservation problems are related to the lack of participation and involvement of rural collectives, initiatives to incorporate participatory processes are rare and usually unsuccessful. It is therefore critical to progress in the search for effective strategies and tools to achieve this objective. In this context, hunting societies in Spain could be a paradigmatic experience of this "bottom-up" conservation model. Hunting societies have an extraordinary potential as a conservation tool since: i) they manage large areas, over 6.5 million hectares, many of high conservation value; ii) they are soundly established in rural areas, and iii) their social model, with a participatory and democratic decision-making system. Despite this, little is known of this exploitation model and of the effects of its environmental externalities. Progress in this field would open a high value, innovative line of research for biodiversity conservation in Europe.

Key words: hunting, stakeholders, communal management, bottom-up conservation.

Las estrategias de conservación "de arriba hacia abajo" se han mostrado insuficientes para frenar la pérdida de biodiversidad

Hasta ahora, la conservación de la biodiversidad en Europa ha seguido por lo general un modelo "de arriba hacia abajo", dirigida desde administraciones y organizaciones conservacionistas, casi siempre basada en una aceptable disposición presupuestaria y, por lo general, con una escasa preocupación en que la toma de decisiones siga un proceso participado y democrático. Los éxitos parciales en la conservación de la biodiversidad de este modelo, no han servido para frenar la pérdida de biodiversidad (Butchart et al. 2010).
Hoy se considera esencial la participación directa de las partes interesadas en los programas de conservación. En esta nueva etapa de la conservación en Europa, es necesario impulsar modelos de conservación "de abajo hacia arriba"

Por el contrario, en los países descentralizados de África, Sudamérica y Asia, hace tiempo que los saberes, usos tradicionales y modelos participativos de las comunidades locales han sido incorporados en las estrategias de conservación, considerando hoy indispensable su participación para el éxito de los programas de conservación (Betancourt Posada 2006; BirdLife International 2010). Sin embargo, en Europa, en donde muchos fracasos en conservación tienen su origen en la escasa participación de los colectivos rurales 
en la toma de decisiones (Reed 2008, Niedziałkowski et al. 2012), las iniciativas para incorporar procesos participativos han sido escasas y generalmente poco exitosas (Rescia et al. 2010, Niedziałkowski et al. 2012). Ante este panorama, existe un interés creciente en incorporar modelos de conservación "de abajo hacia arriba". El empoderamiento y la participación efectiva de la sociedad en la conservación de la biodiversidad y especialmente de las comunidades locales, son ahora objetivos específicos en las estrategias de conservación en gobiernos y ONG (ver p.e. el Plan Estratégico para la Diversidad Biológica 2011-2020 y las metas de Aichi o la estrategia de la UE sobre biodiversidad hasta 2020). Además, la necesidad de esta nueva estrategia se ve reforzada en el contexto actual de crisis económica y la subsiguiente escasez de fondos públicos para la conservación. Este reto ha suscitado un debate sobre qué procesos y herramientas serían las más adecuadas para conseguir una participación real y efectiva de los colectivos rurales que viven en y de los territorios donde se instauran los programas de conservación (Stringer et al. 2006), siendo preciso crear y mejorar herramientas y metodologías en los procesos de participación (Reed 2008).

Las sociedades de cazadores en España podrían suponer una experiencia paradigmática de este modelo de conservación de "abajo hacia arriba"

En este nuevo enfoque, las sociedades de cazadores (SC) podrían desempeñar un importante papel en la conservación de la biodiversidad. Las SC tutelan en España uno de los mayores aprovechamientos comunales de toda Europa, no sólo en cuanto a la gran superficie sobre las que tienen derecho de gestión, un mínimo de 6.5 millones de ha, sino también en cuanto al número de personas que participan en los cuerpos de gobernanza de sus derechos cinegéticos(Couto y Gutiérrez 2012). Sólo las SC federadas tienen más de 360000 cazadores asociados (Consejo superior de Deportes 2012) por lo que el número total de socios debe ser sensiblemente superior (no todas las SC están federadas). Aparte de esta relevancia territorial y social, la pertenencia a la comunidad, el autogobierno y la participación democrática en la toma de decisiones son cualidades que cualifican especialmente a las SC para seguir iniciativas de conservación "de abajo hacia arriba". Estas sociedades se amparan en el artículo 22 de la Constitución Española y tienen su respaldo legal en la Ley Orgánica 1/2002, de 22 de Marzo, reguladora del Derecho de Asociación, o bien en decretos autonómicos para la constitución de entidades deportivas sin ánimo de lucro. En ambos casos, el órgano máximo de decisión es la Asamblea General de Socios y, aunque es necesario avanzar en la calidad de los procesos participativos (sería deseable una implicación más activa y efectiva de los socios para aprovechar esta cualidad esencial en las SC), el funcionamiento democrático está legalmente garantizado. Además, al contrario que otros aprovechamientos comunales, que perdieron paulatinamente reconocimiento legal, las SC lograron un gran respaldo legislativo e institucional con la Ley de Caza de 1970 que se ha mantenido en sucesivas leyes estatales y autonómicas hasta la actualidad (Couto y Gutiérrez 2012), siendo este tipo de aprovechamiento cinegético el modelo mayoritario en muchas zonas de España (Delibes-Mateos y Arroyo 2012).

Por tanto, la gran superficie sobre las que actúan (buena parte con un alto valor de conservación), la relación de sus actividades con la conservación de la biodiversidad, su implantación en el medio rural, su modelo social y su vocación participativa y democrática en la toma de decisiones, las dota en su conjunto de un extraordinario potencial como herramienta de conservación. Sin embargo este potencial ha pasado hasta ahora prácticamente desapercibido $y$, aunque recientemente existen experiencias positivas en nuestro país que demuestran la predisposición y las capacidades que tienen las SC para trabajar en conservación (Martínez et al. 2010, Fundación Gypaetus 2010, Gutiérrez 2010), es necesario que investigadores, administraciones, ONG y las propias SC tomen una mayor conciencia de este potencial para frenar la pérdida de biodiversidad en el medio rural.
Apenas se dispone de información adecuada sobre el balance de las externalidades ambientales de las SC, pero la ausencia de criterios técnicos en la gestión cinegética en la mayoría de ellas y la falta de compromiso real con la conservación de la biodiversidad, han sido hasta ahora importantes limitaciones

La información disponible sobre las SC es muy escasa. Por ejemplo, falta información detallada sobre la superficie que gestionan, el número de personas que aglutinan o la cantidad de recursos económicos que destinan a la vigilancia y mejora de sus terrenos de caza. En cuanto a sus externalidades ambientales, apenas existe información que permita una evaluación diferenciada de la aportación de las SC a la conservación de los recursos cinegéticos y de la biodiversidad en general. Es innegable que la falta generalizada de criterios técnicos en la gestión cinegética y los escasos avances en este sentido no han permitido, en la mayoría de los casos, una gestión adecuada de los recursos cinegéticos. Más bien al contrario, el modelo obsoleto de aprovechamiento con cupos fijos y las autolimitaciones en los periodos de caza que, como principales medidas de gestión, predominan en la mayoría de las SC, no ha servido para frenar en muchos casos el declive en las poblaciones de especies cinegéticas de sus acotados. Además, en demasiadas ocasiones, actuaciones y recetas intuitivas sin base científico-técnica, han tenido consecuencias indeseables en poblaciones cinegéticas y no cinegéticas (por ejemplo, repoblaciones sistemáticas o actuaciones para el control de predadores) (Arroyo y Beja 2002). Por otro lado, en España, al contrario que en otros países europeos, ni las SC, ni las organizaciones que las representan han adoptado aún una apuesta decidida, comprometida y participativa en la conservación de la biodiversidad.

Sin embargo, su modelo social y el creciente interés de las SC y de las organizaciones que las representan en mejorar la gestión cinegética y participar en la conservación de la biodiversidad, suponen un buen punto de partida

Pero también es cierto que el modelo de organización social de las SC tiene efectos positivos sobre la conservación. Por un lado, la resiliencia de las SC basada en los mecanismos de autodefensa de sus intereses colectivos, ha supuesto un freno a la expansión de la caza comercial y a los conflictos que genera la intensificación de la caza en la conservación de la biodiversidad (García 1986, Arroyo et al. 2012, Couto y Gutiérrez 2012). Por otro lado, los efectos negativos del abandono rural para la conservación de la biodiversidad (Moreira and Russo 2007), han sido atenuados en parte por la cohesión social que induce la existencia de estas asociaciones. También es destacable que en los últimos años se haya acrecentado el interés por la mejora de la gestión cinegética por parte de la Real Federación Española de Caza (RFEC) y sus federaciones autonómicas, a las que están asociadas la mayoría de SC. En cuanto al compromiso real con la conservación de la biodiversidad, en la actualidad existen determinadas asociaciones que también aglutinan a las SC que están divulgando y potenciando su participación en la conservación de la biodiverisdad. En este sentido es destacable la labor de la Unión Nacional de Asociaciones de Caza (UNAC), recientemente registrada como ONG medioambiental y que, entre otras medidas, impulsa la participación de las SC en iniciativas de custodia del territorio. Por último, las SC comienzan a incorporar la conservación de la biodiversidad entre sus objetivos estatutarios (ver por ejemplo SC "La Laurisilva", http://laurisilvasociedadcazadoresgmailcom.blogspot.com.es/p/estatutos.html).

Con su apuesta decidida por la gestión cinegética de calidad, la participación activa en las estrategias de conservación y con una mayor atención y apoyo por parte de administraciones, instituciones científicas y ONG conservacionistas, las SC podrían convertirse en inestimables aliados para la conservación de la biodiversidad

Hace falta, por tanto que, por un lado las SC inviertan esfuerzos y recursos en incorporar los conocimientos científicos y técnicos en la gestión sus acotados (p.e, siguiendo el ejemplo de Francia, en 
donde la gestión cinegética está mucho más tecnificada). Y por otro lado establecer líneas de trabajo e investigación que establezcan qué modelos y actuaciones podrían incorporar con facilidad las SC en sus actividades de gestión para contribuir a la conservación de la biodiversidad. El conocimiento de estos modelos y herramientas y la inclusión voluntaria de objetivos de sostenibilidad y fomento de la biodiversidad en sus estatutos y normas reguladoras, podrían convertir a las SC en un ejemplo paradigmático de conservación de "abajo hacia arriba".

\section{Referencias}

Arroyo, B., Beja, P. 2002. Impact of hunting management practices on biodiversity. Report WP2 to REGHAB project. European Commission, Brussels. Belgium. Disponible en: http://digital.csic.es/bitstream/10261/8260/1/WP2-repportf.PDF

Arroyo, B. Delibes-Mateos, M. Díaz-Fernández, Viñuela, J. 2012. Hunting management in relation to profitability aims: red-legged partridge hunting in central Spain. European Journal of Wildlife Research 58:847-855.

BirdLife International 2010. Conservación de la Biodiversidad y Comunidades Locales. BirdLife International. Quito, Ecuador: Disponible en: http://www.birdlife.org/downloads/nagoya/Biodiversity-conservationlocal-communities-es.pdf

Betancourt Posada, A. (coord.) 2006. De la conservación desde arriba a la conservación desde abajo: el interés supranacional en los saberes indígenas sobre ecología, Fundación Carolina CeALCI 22/05, pp. 9-37. Madrid, España.

Butchart, S.H.M., Walpole, M., Collen, B., van Strien, J.P.W., Scharlemann, R.E.A., Almond, J.E.M. et al. 2010. 'Global biodiversity: indicators of recent declines. Science 328:1164-1168.

Consejo Superior de Deportes 2012. Memoria 2012/Licencias y Clubes. Consejo Superior de Deportes. Gobierno de España. Disponible en : http://www.csd.gob.es/csd/estaticos/asoc-fed/licenciasyclubes-2012.pdf

Couto, S., Gutiérrez, J.E., 2012. Recognition and Support of ICCAs in Spain En: Kothari, A., Corrigan, C., Jonas, H., Neumann, A., Shrumm, H. (eds). Recognising and Supporting Territories and Areas Conserved By Indigenous Peoples And Local Communities: Global Overview and National Case Studies. Technical Series no. 64. Secretariat of the Convention on Biological Diversity, ICCA Consortium, Kalpavriksh, and Natural Justice, Montreal, Canada. Disponible en:

http://www.cbd.int/pa/doc/ts64-case-studies/spain-en.pdf

Delibes-Mateos, M., Arroyo, B. (Eds.). 2012. Hunting for Sustainability: a summary of research findings from Spain. Seventh Framework Programm and European Commission. Instituto de Investigación en Recursos Cinegéticos. UCLM. Ciudad Real, España.

Fundación Gypaetus 2010. LIFE04 NAT/ES/000056. Laymant report. Disponible en:

http://ec.europa.eu/environment/life/project/Projects/index.cfm?fuseaction=search.dspPage\&n proj id $=2604$

García, F. 1986. Las sociedades de cazadores en Asturias. Un ejemplo de las estrategias para la defensa del espacio rural de la invasión urbana. Ería 10:143-145

Gutiérrez, J.E. 2010. Sin bajar la guardia. Identificación y control de amenazas, un puente con el mundo rural. Quercus 283 (edición especial: El Quebrantahuesos de nuevo en Andalucía): 22-29.

Martínez, E. Sánchez, J., Santos, R. 2010. Caza y Custodia de territorio en los paisajes agrarios españoles. En: Leco Berrocal, F. (coord.). Actas del XV Coloquio de Geografía Rural: Territorio, paisaje y patrimonio rural, 28 a 30 de abril de 2010. Cáceres. Servicio de publicaciones Universidad de Extremadura. España.

Moreira, F., Russo, D. 2007. Modelling the impact of agricultural abandonment and wildfires on vertebrate diversity in Mediterranean Europe. Landscape Ecology 22:1461-1476.

Niedziałkowski, K., Paavola, P., Jędrzejewska, B. 2012. Participation and protected areas governance: the impact of changing influence of local authorities on the conservation of the Białowieża Primeval Forest, Poland. Ecology and Society 17(1):2.

Reed, M.S. 2008. Stakeholder participation for environmental management: A literature review. Biological Conservation 141(10):2417-2431.

Rescia, A.J., Fungairiño, S.G., Dover J.W. 2010. Reactivación del sistema socioecológico ganadero de Picos de Europa (norte de España). Ecosistemas 19(2):137-145.

Stringer, L.C., Dougill, A.J., Fraser, E., Hubacek, K., Prell, C., Reed, M.S. 2006. Unpacking "participation" in the adaptive management of socialecological systems: a critical review. Ecology and Society 11(2):39. 